\title{
ADDENDUM TO "SOME CASES OF VOJTA'S CONJECTURE ON INTEGRAL POINTS OVER FUNCTION FIELDS", JOURNAL ALG. GEOMETRY, VOL. 17, 295-333, 2008*
}

PIETRO CORVAJA ${ }^{\dagger}$ AND UMBERTO ZANNIER ${ }^{\ddagger}$

This note is an Addendum to our paper published in Journal Alg. Geometry, 17 (2008), 295-333, indicated throughout as [CZ]. It has two main purposes:

(i) Adding a few details to clarify a certain step in the proof of Theorem 1.2 in [CZ]; our treatment of Lemma 3.14 was not comprehensive of the most general case.

(ii) Adding a further counterexample to the ones in $\S 4$, completing the analysis of the integral points for the complement of a cubic in the plane.

We take this opportunity to include as well a short

(iii) Errata - corrige.

We have also decided to append in the arxiv (http://arxiv.org/abs/math/0512074 v3) a completely rewritten version of the paper [CZ], which the reader may find convenient to consult.

$\S(\mathrm{i})$. In Lemma 3.14 the assumptions on $\alpha, \beta$ should be that $A(\alpha, \beta)=B(\alpha, \beta)=$ 0 . We observe that, with this assumption, the proof works as it stands if $(\alpha, \beta)$ is non singular on the curve $A(X, Y)=0$; on the other hand, any singular point would be defined over $\kappa$, like the polynomial $A$, and Lemma 3.14 would be anyway automatic.)

On the contrary, in [CZ] we only imposed that $F(\alpha)=G(\beta)=0$ for the resultants $F(X), G(Y)$ of $A(X, Y), B(X, Y)$ with respect to $Y, X$.

In general, this last assumption does not imply the previous one. However such implication holds in our situation, due to the actual shape of the polynomials $A, B$ (given by (3.6) of page 312). In fact, the formulas of page 313, lines $-13 /-11$, yield $F(X)=c_{1} A(X, Y)-B(X, Y), G(Y)=c_{2} A(X, Y)+c_{3} B(X, Y)$ for certain $c_{1}, c_{2}, c_{3} \in \kappa(\tilde{\mathcal{C}})$. Now, we have $c_{1} c_{3}+c_{2} \neq 0$, for otherwise $F(X), G(Y)$ would be linearly dependent over $\kappa(\tilde{\mathcal{C}})$, and thus would neither depend on $X$ nor on $Y$, which is impossible (looking at their coefficients we would find that $u_{1}, u_{2}$ are both constant). Then the vanishing of $F(\alpha)$ and $G(\beta)$ implies indeed $A(\alpha, \beta)=B(\alpha, \beta)=0$, as required.

In conclusion, Lemma 3.14 is alright as it stands concerning the proof of Theorem 1.1. However, we have to modify it as indicated above for the application to the proof of Theorem 1.2, through Proposition 3.16; in fact the argument just given does not apply in the general case, especially at page 326 , line 5 . We now indicate how to perform the same application, starting from the new version of the lemma.

So, in the proof of Proposition 3.16, we need to restrict the pairs $(\alpha, \beta)$ to the set $\mathcal{Z}:=\left\{(\alpha, \beta) \in \kappa(\tilde{\mathcal{D}})^{2} \mid \alpha \neq 0, \beta \neq 0, A(\alpha, \beta)=B(\alpha, \beta)=0\right\}$ in place of $\mathcal{Z}^{\prime}:=$ $\{(\alpha, \beta) \mid F(\alpha)=G(\beta)=0\}$.

*Received June 23, 2010; accepted for publication October 7, 2010.

†Dipartimento di Matematica e Informatica, Via delle Scienze, 206, 33100 - Udine, Italy (corvaja @dimi.uniud.it).

‡Scuola Normale Superiore, Piazza dei Cavalieri, 7, 56100 Pisa, Italy (u.zannier@sns.it). 
Inspection of the proof shows that we need just an inequality of the following shape: for a fixed $v \in \tilde{\mathcal{D}} \backslash U$,

(1) $\min \left(v\left(A\left(u_{1}, u_{2}\right)\right), v\left(B\left(u_{1}, u_{2}\right)\right)\right) \leq m n \cdot \max _{(\alpha, \beta) \in \mathcal{Z}} \min \left(v\left(u_{1}-\alpha\right), v\left(u_{2}-\beta\right)\right)$.

We now prove this inequality. We can plainly disregard the points $(\alpha, \beta)$ with $\alpha \beta=0$, because in that case $\min \left(v\left(u_{1}-\alpha\right), v\left(u_{2}-\beta\right)\right)=0$. Then, to prove the inequality (1), let us first enlarge the finite set $U$ so to contain the zeros of the fixed differential form $\omega$ and so that the (nonzero) values $A(\alpha, \beta), B(\alpha, \beta)$ for $(\alpha, \beta) \in \mathcal{Z}^{\prime} \backslash \mathcal{Z}$ are $U$-units.

Note that this enlargement is harmless for our purposes. In fact, by the height estimates already carried out in the proof (see for instance Step 2 and Step 3, pages 324-325) the heights and degrees of $F(X), G(Y)$ are bounded by a constant time $\max \{1, \chi(\tilde{\mathcal{C}} \backslash S)\}$; therefore the heights of $\alpha, \beta, A(\alpha, \beta), B(\alpha, \beta)$ are likewise bounded. In turn, this implies that the nonzero elements $A(\alpha, \beta)$ in this set have zeros and poles in a new set, still denoted by $U$, obtained by adding at most $\gamma \max (1, \chi(\tilde{\mathcal{C}} \backslash S))$ new points, for a suitable constant $\gamma$ depending only on $m, n$.

Now, $F(X), G(Y)$ are linear combinations of $A, B$ with $U$-integral coefficients, so for $v$ out of $U$,

$$
\min \left\{v\left(A\left(u_{1}, u_{2}\right), v\left(B\left(u_{1}, u_{2}\right)\right)\right\} \leq \min \left\{v\left(F\left(u_{1}\right)\right), v\left(G\left(u_{2}\right)\right)\right\}\right.
$$

By the inequality $\min \left(\sum a_{i}, \sum b_{j}\right) \leq \sum_{i, j} \min \left(a_{i}, b_{j}\right)$ holding for arbitrary $a_{i}, b_{j} \geq 0$ (which expresses that $\operatorname{gcd}(x, y)$ is sub-multiplicative in both variables), we infer

$$
\min \left\{v\left(A\left(u_{1}, u_{2}\right), v\left(B\left(u_{1}, u_{2}\right)\right)\right\} \leq \sum_{i, j} \min \left\{v\left(u_{1}-\alpha_{i}\right), v\left(u_{2}-\beta_{j}\right)\right\}\right.
$$

This is inequality (1) to be proved, but with $\mathcal{Z}^{\prime}$ in place of $\mathcal{Z}$. To deduce (1) from this, let $v \in \tilde{\mathcal{D}} \backslash U$ and suppose that the minimum on the left in (1) is positive. Then, we assert that the maximum $\max _{(\alpha, \beta) \in \mathcal{Z}^{\prime}} \min \left(v\left(u_{1}-\alpha\right), v\left(u_{2}-\beta\right)\right)$ cannot be attained at a point $(\alpha, \beta) \in \mathcal{Z}^{\prime} \backslash \mathcal{Z}$. In fact, if this were the case, by (2) we get

$$
0<\min \left(v\left(A\left(u_{1}, u_{2}\right)\right), v\left(B\left(u_{1}, u_{2}\right)\right)\right) \leq m n \cdot \min \left(v\left(u_{1}-\alpha\right), v\left(u_{2}-\beta\right)\right)
$$

Now, since $(\alpha, \beta) \in \mathcal{Z}^{\prime} \backslash \mathcal{Z}$, by definition at least one between $A(\alpha, \beta), B(\alpha, \beta)$ is non-zero, say $A(\alpha, \beta) \neq 0$; by the present choice of $U$, we deduce that $A(\alpha, \beta)$ is a $U$-unit, so in particular $v(A(\alpha, \beta))=0$. On the other hand, $v\left(u_{1}-\alpha\right)$ and $v\left(u_{2}-\beta\right)$ are $>0$, (because of the above inequality) hence $v\left(A\left(u_{1}, u_{2}\right)\right)=0$ as well (since $\left.A(\alpha, \beta) \equiv A\left(u_{1}, u_{2}\right) \quad(\bmod v)\right)$.

This is a contradiction, and therefore the maximum is attained in $\mathcal{Z}$, so (1) is proved.

We finally remark that, anyway, the role of Lemma 3.14 is essentially of brevity and simplicity of formulation of some theorems; in fact, even forgetting Lemma 3.14, the rest of the proof produces a non-trivial multiplicative relation $u_{1}^{r} u_{2}^{s}=\mu$, with bounded exponents $r, s$ and $\mu \in \kappa(\tilde{\mathcal{C}})^{*}$ of bounded height. After an automorphism of $\mathbf{G}_{m}^{2}$ we may thus suppose that $u_{1}$ itself has bounded height, and thus belongs to a finite set up to constants. This reduces to a problem where only $u_{2}$ varies, which can be easily dealt by the abc-theorem. 
$\S($ ii). We complete the discussion of $\S 4$ in [CZ] about the complement of a cubic on the plane. In $[\mathrm{CZ}]$ we already proved that if $D$ is a reducible cubic, then the surface $X:=\mathbf{P}_{2} \backslash D$ is not algebraically hyperbolic, meaning that no bound of the form (1.1) holds for curves on $X$. We now treat the case of an irreducible cubic $D$, by proving the following

Theorem. Let $D \subset \mathbf{P}_{2}$ be an irreducible cubic. For every integer $d \geq 1$ there exists a curve $Y \subset X:=\mathbf{P}_{2} \backslash D$ such that $\operatorname{deg} Y \geq d$ and $\chi(Y) \leq 12$.

We start with the easiest cases of singular cubics.

Consider first the case when $D$ has a cusp. Then in suitable coordinates $D$ is given by the equation $D: z y^{2}-x^{3}=0$. Given a polynomial $p(t) \in \kappa[t]$, with $p(0) \neq 0$, and a positive integer $n$, the map $\mathbf{G}_{m} \rightarrow \mathbf{P}_{2}$ sending $\mathbf{G}_{m} \ni t \mapsto\left(t^{2 n} p(t): t^{3 n}: p(t)^{3}+1\right)$ avoids the divisor $D$ and its image is a curve of degree $\geq 2 n+\operatorname{deg} p(t)$. We then obtain curves of arbitrarily large degree and vanishing Euler characteristic, as in Theorems $4.1,4.2$ of $[\mathrm{CZ}]$.

Let now $D$ be a nodal irreducible cubic; in some coordinates it takes the equation $z y^{2}=x^{3}+x^{2} z$. For every integer $n>0$, let us define the morphism $\mathbf{G}_{m} \rightarrow \mathbf{P}_{2}$ by sending

$$
\mathbf{G}_{m} \ni t \mapsto\left(4 t(t-1): 4 t(t+1):(t-1)^{3}+t^{n}\right)
$$

Again, its image avoids the curve $D$, obtaining the same conclusion as in the cuspidal case.

We now come to the most interesting case where $D$ is smooth. We shall produce curves of Euler characteristic $\leq 12$ and of arbitrarily high degree. We let $P_{1}, \ldots, P_{9}$ be the flexi of $D$, and $P_{10}, P_{11}, P_{12}$ the three points ( $P_{1}$ apart) whose tangent passes through $P_{1}$. In other words, if $P_{1}$ is taken as the origin of the elliptic curve corresponding to $D$, then $P_{1}, \ldots, P_{9}$ are the points of three-torsion and $P_{10}, P_{11}, P_{12}$ are the points of exact order two.

Put $S=\left\{P_{1}, \ldots, P_{12}\right\}$ and $\mathcal{C}:=D \backslash S$; since $D$ has genus one, $\mathcal{C}$ is an affine curve with $\chi(\mathcal{C})=12$.

Finally, let $u \in \mathcal{O}_{S}^{*}$ be a non constant $S$-unit; note that $u$ exists and actually $\mathcal{O}_{S}^{*} / \kappa^{*}$ has rank eleven: in fact any point $P_{i}, 1<i \leq 12$, is torsion with respect to $P_{1}$ of order 2 or 3 , so there are functions $f_{i}$ with divisors $\left(f_{i}\right)=6\left(P_{1}-P_{i}\right)$.

For every point $p \in D \backslash S$, the tangent $T_{p}$ at $D$ in $p$ will intersect the cubic $D$ at a second point $p^{\prime}$, which is distinct from $p$, since $p$ is not a flexus. Also, $T_{p}$ will intersect the tangent line $T_{P_{1}}$ at $P_{1}$ at a point $p^{\prime \prime}$ distinct from $P_{1}$, since $p \notin\left\{P_{10}, P_{11}, P_{12}\right\}$. The point $p^{\prime \prime}$ does not lie on $D$, since $T_{P_{1}} \cap D=\left\{P_{1}\right\}$.

We can canonically identify the line $T_{p}$ with $\mathbf{P}_{1}$ in such a way that $p$ corresponds to the point at infinity, $p^{\prime}$ to 0 and $p^{\prime \prime}$ to 1 .

We define a morphism $\mathcal{C} \rightarrow \mathbf{P}_{2}$ by sending $p$ to the point $\varphi(p) \in T_{p}$, where $\varphi(p)$ is the point of coordinate $u(p)$, in the above coordinate system.

By construction, $\varphi(p)$ never lies on $D$ (otherwise $u(p)=0$ or $\infty$, which does not happen on $\mathcal{C}$ ).

The image $\varphi(\mathcal{C})$ is a curve of degree $\geq \operatorname{deg}(u)$ : in fact, $\varphi(\mathcal{C})$ intersects $T_{P_{1}}$ whenever $u(p)=1$ hence the intersection $\varphi(\mathcal{C}) \cap T_{P_{1}}$ has at least $\operatorname{deg} u$ points, so $\operatorname{deg} \overline{\varphi(\mathcal{C})} \geq \operatorname{deg} u$. Since $\operatorname{deg} u$ may be taken arbitrarily large (for instance replacing $u$ with $u^{n}$ ) one obtains curves parametrized by $\mathcal{C}$ of arbitrarily high degree.

The result just proved is in accordance with its arithmetic analogue, proved by Beukers in the reference [Be] of [CZ], stating that the integral points on the comple- 
ment of a smooth cubic curve on $\mathbf{P}_{2}$ are Zariski dense over a suitable ring of $S$-integers. Its complex-analytic analogue also holds, actually in stronger form: a recent theorem of Buzzard and $\mathrm{Lu}$ [Algebraic surfaces holomorphically dominable by $\mathbf{C}^{2}$, Inv. Math . 139 (2000), 617-659] provides the existence of a map $\mathbf{C}^{2} \rightarrow \mathbf{P}_{2} \backslash D$ whose differential has generically rank two; in particular, one obtains Zariski dense entire curves in the plane omitting the cubic $D$.

$\S($ iii). Errata - corrige.

(1) At page 303 and in the subsequent part of $\S 2$, the symbol $K$ denotes the function field $\kappa(\tilde{\mathcal{C}})$. This notation has been abandoned in the subsequent $\S 3$, since we needed to work with different function fields $(\kappa(\tilde{\mathcal{C}}), \kappa(\tilde{\mathcal{D}}), \ldots)$.

(2) Page 312, Lemma 3.7. In the displayed formula, the last factor should be $\frac{\partial A}{\partial Y}(X, Y)$ in place of $\frac{\partial B}{\partial Y}(X, Y)$.

(3) Page 313, formula (3.7). In the definition of $G(Y)$, on the right side of the inequality, the monomials $X^{2}, X$ should be $Y^{2}, Y$.

(4) Page 315 , line 8: $\chi_{S}(\tilde{\mathcal{C}})$ should be $\chi_{S \cup T}(\tilde{\mathcal{C}})$.

(5) Page 323, line -9: there should be then.

(6) Page 326, line 2: $\min \left\{v\left(u_{1}-\alpha_{i}\right), v\left(u_{2}-\beta_{j}\right)\right\}$, not $\min \left\{v\left(u_{1}-\alpha_{1}\right), v\left(u_{2}-\beta_{2}\right)\right\}$.

(7) Page 232 , lines $-5,-3$. The omission of a parenthesis makes the sentence obscure. Read: "considering two different monomials (if $A(X, Y)$ were a monomial, the function $A\left(u_{1}, u_{2}\right)$ would have no zero outside $S$ and (3.23) would be trivial) we obtain...".

Acknowledgements. We are pleased to thank Prof. S. Yau for his interest in our paper and an anonymous referee for his technical comments. 